Article

\title{
Synthesis and QSAR Study of Novel 6-Chloro-3-(2- Arylmethylene-1-methylhydrazino)-1,4,2-benzodithiazine 1,1-Dioxide Derivatives with Anticancer Activity
}

\section{Jarosław Slawiński 1,*, Beata Żolnowska ${ }^{1}$, Zdzisław Brzozowski ${ }^{1}$, Anna Kawiak ${ }^{2,3}$, Mariusz Belka ${ }^{4}$ and Tomasz Bączek ${ }^{4}$}

1 Department of Organic Chemistry, Medical University of Gdańsk, Al. Gen. J. Hallera 107, 80-416 Gdańsk, Poland; E-Mails: zolnowska@gumed.edu.pl (B.Ż.);

brzozowskigumed@gmail.com (Z.B.)

2 Department of Biotechnology, Intercollegiate Faculty of Biotechnology, University of Gdańsk and Medical University of Gdańsk, ul. Kładki 24, 80-822 Gdańsk, Poland; E-Mail: kawiak@biotech.ug.pl

3 Department of Human Physiology, Medical University of Gdańsk, ul. Tuwima 15, 80-210 Gdańsk, Poland

4 Department of Pharmaceutical Chemistry, Medical University of Gdańsk, A1. Gen. J. Hallera 107, 80-416 Gdańsk, Poland; E-Mails: mariusz.belka@gumed.edu.pl (M.B.); tbaczek@gumed.edu.pl (T.B.)

* Author to whom correspondence should be addressed; E-Mail: jaroslaw@gumed.edu.pl; Tel.: +48-58-349-1098; Fax: +48-58-349-1277.

Academic Editor: Derek J. McPhee

Received: 24 February 2015 / Accepted: 26 March 2015 / Published: 1 April 2015

\begin{abstract}
A series of new 6-chloro-3-(2-arylmethylene-1-methylhydrazino)-1,4,2benzodithiazine 1,1-dioxide derivatives were effectively synthesized from $N$-methyl- $N$-(6chloro-1,1-dioxo-1,4,2-benzodithiazin-3-yl)hydrazines. The intermediate compounds as well as the products, were evaluated for their cytotoxic effects toward three human cancer cell lines. All compounds shown moderate or weak cytotoxic effects against the tested cancer cell lines, but selective cytotoxic effects were observed. Compound 16 exhibited the most potent cytotoxic activity against the HeLa cell line, with an IC50 value of $10 \mu \mathrm{M}$, while 14 was the most active against the MCF-7 and HCT-116 cell lines, affording IC50 values of $15 \mu \mathrm{M}$ and $16 \mu \mathrm{M}$, respectively. The structure-activity relationship was evaluated based on QSAR methodology. The QSAR MCF-7 model indicated that natural charge on carbon atom
\end{abstract}


C13 and energy of highest occupied molecular orbital (HOMO) are highly involved in cytotoxic activity against MCF-7 cell line. The cytotoxic activity of compounds against HCT-116 cell line is dependent on natural charge on carbon atom C13 and electrostatic charge on nitrogen atom N10. The obtained QSAR models could provide guidelines for further development of novel anticancer agents.

Keywords: sulfonamides; 1,4,2-benzodithiazines; cytotoxic activity; anticancer activity; structure-activity relationship; QSAR; stepwise regression

\section{Introduction}

The 1,1-dioxo-1,4,2-benzodithiazines constitute a fundamental class of compounds in our laboratories since 1984, they have a wide range of biological activity and are useful substrates for many syntheses. It has been demonstrated that many 6-chloro-1,1-dioxo-1,4,2-benzodithiazine derivatives (I, Figure 1) possess low acute toxicity in mice and rats and depending on their structure, they act as potential diuretic [1-6] radioprotective [4], cholagogue [6,7], antiarrhythmic [4,6], or hypotensive [4,5-7] agents. It has also been shown that some 6-chloro-1,1-dioxo-1,4,2-benzodithiazines exhibit remarkable antitumor activities (I [8-10], II and III [10-12], Figure 1) or anti-HIV-1 activity (I and IV [13-15] Figure 1). This prompted us to investigate new potential antiproliferative agents with the general structure of type $\mathbf{V}$ (Figure 1) in which moieties attached to positions 3 and 7 of 6-chloro-1,1-dioxo-1,4,2benzodithiazine ring were varied in molecular shape and electronic properties.

Thus, we synthesized new series of 6-chloro-7-R -3 -(2-arylmethylene-1-methylhydrazino)-1,1dioxo-1,4,2-benzodithiazines $\mathbf{V}$ and evaluated their in vitro anticancer activity against human breast (MCF-7), colon (HCT-116) and cervical (HeLa) cancer cell lines. To explain how structural features influence the biological activities the quantitative structure-activity relationship (QSAR) method was applied [16].<smiles>[R]C1=NS(=O)(=O)c2cc(Cl)c(Cl)cc2S1</smiles>

I<smiles>[R]c1cc2c(cc1Cl)Sc1ncc([R])n1S2(=O)=O</smiles>

II<smiles>[R]c1nc2n(n1)S(=O)(=O)c1cc(Cl)c(Cl)cc1S2</smiles>

III<smiles>[R]c1c(Cl)cc2c(c1[R])S(=O)(=O)NC(C(=O)[Al])S2</smiles>

IV<smiles>[R]c1cc2c(cc1Cl)SC(N(C)N=C[Al])=NS2(=O)=O</smiles>

v

Figure 1. Chemical structures of biological active 1,1-dioxo-1,4,2-benzodithiazines I-IV [1-15], and V. 


\section{Results and Discussion}

\subsection{Chemistry}

A series of the desired 6-chloro-7-R ${ }^{1}$-3-(2-arylmethylene-1-methylhydrazino)-1,1-dioxo-1,4,2benzodithiazine derivatives 5-18 was prepared in a two-step process as shown in Schemes 1 and 2. Thus, the starting $N$-methyl- $N$-(6-chloro-7-R ${ }^{1}$-1,1-dioxo-1,4,2-benzodithiazin-3-yl)hydrazines $2-\mathbf{4}$ were synthesized by the reaction of the appropriate 3-methylthiobenzodithiazines 1a-c with $N$-methylhydrazine in boiling dry acetonitrile (Scheme 1). It is well known that alkylations of alkyl-substituted hydrazines take place at the more substituted nitrogen atom [17-19], therefore in our investigations competition between $N^{1}$ and $N^{2}$ alkylation was not observed, and the regioselective substitution product, i.e., $N$-methyl- $N$-substituted hydrazines, were obtanined in high yields (90\%-93\%). Subsequent condensation of the hydrazine derivatives with an aldehyde in the presence of a catalytic amount of glacial acetic acid furnished the expected final products 5-18 in good yields (87\%-98\%) (Scheme 2).
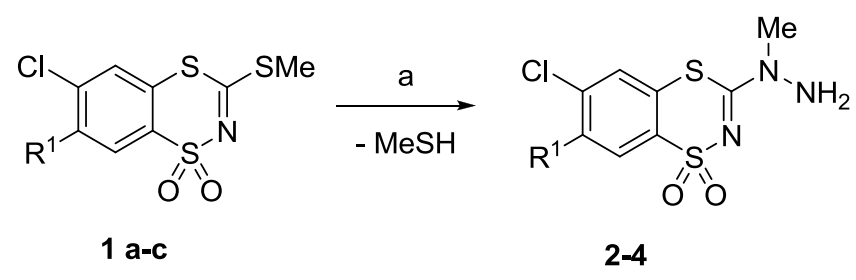

\begin{tabular}{lll} 
Compd. & $\mathrm{R}^{1}$ & Yield \% \\
\hline $\mathbf{1 a}, \mathbf{2}$ & $\mathrm{Me}$ & 93 \\
$\mathbf{1 b}, \mathbf{3}$ & MeOCO & 90 \\
$\mathbf{1 c}, \mathbf{4}$ & $\mathrm{NC}$ & 91
\end{tabular}

Reagents and conditions: (a) methylhydrazine (1.04 M equiv.), dry acetonitrile, r.t. 8 h, reflux 10-16 h.

Scheme 1. Synthesis of $N$-methyl- $N$-(6-chloro-1,1-dioxo-1,4,2-benzodithiazin-3-yl)hydrazines 2-4.

The structures of the compounds $\mathbf{2}-\mathbf{4}$ and the final substances 5-18 were confirmed by IR, ${ }^{1} \mathrm{H}-\mathrm{NMR}$ and ${ }^{13} \mathrm{C}-\mathrm{NMR}$ spectroscopy. For example, in the ${ }^{1} \mathrm{H}-\mathrm{NMR}$ spectra, the presence of $\mathrm{N}-\mathrm{CH}_{3}$ and $\mathrm{N}-\mathrm{NH}_{2}$ groups in 2-4 was identified from the singlet signals at 3.36-3.35 and 5.72-5.85 ppm, respectively. Meanwhile, the appearance of $\mathrm{N}=\mathrm{CH}$ signals at $8.21-8.66 \mathrm{ppm}$ in the spectra of $\mathbf{5}-\mathbf{1 8}$ proved the presence of this group and conformed the proposed structure of the final compounds.

\subsection{Cytotoxic Activity}

Compounds 6-18 and the intermediates $\mathbf{2}$ and $\mathbf{4}$ were evaluated in vitro for their effects on the viability of three human cancer cell lines: MCF-7 (breast cancer), HCT-116 (colon cancer) and HeLa (cervical cancer). The concentration required for $50 \%$ inhibition of cell viability $\mathrm{IC}_{50}$ was calculated and compared with the reference drug cisplatin, and the results are given in Table 1.

The most active compounds possess at $N^{2}$ of the 3-hydrazinobenzodithiazine scaffold a 2,4-dihydroxyphenyl (compound 6) or 2-hydroxy-5-nitrophenyl (compound 9) moiety and showed outstanding anticancer activity against all tested cell lines, whereas compounds 2, 4, 7, 13 and 18 demonstrated relatively weak activity, with $\mathrm{IC}_{50}$ values over $100 \mu \mathrm{M}$. 
<smiles>[R]c1cc2c(cc1Cl)SC(N(C)N)=NS2(=O)=O</smiles>

2-4

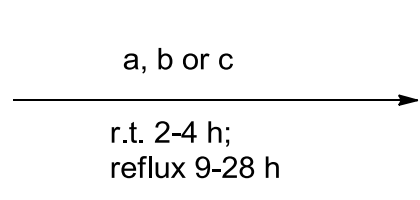

reflux $9-28 \mathrm{~h}$<smiles>[R]c1cc2c(cc1Cl)SC(N(C)/N=C/[Al])=NS2(=O)=O</smiles>

$5-18$

\begin{tabular}{|c|c|c|c|}
\hline Compd. & Method & $\mathrm{R}^{1}$ & $\mathrm{Ar}$ \\
\hline 2,5 & $a$ & $\mathrm{Me}$ & $\mathrm{HC}$ \\
\hline 2,6 & $a$ & $\mathrm{Me}$ & \\
\hline 2,7 & $a$ & $\mathrm{Me}$ & \\
\hline 2,8 & $a$ & $\mathrm{Me}$ & \\
\hline 2,9 & $a$ & $\mathrm{Me}$ & \\
\hline 2,10 & $a$ & $\mathrm{Me}$ & \\
\hline 2,11 & $a$ & $\mathrm{Me}$ & \\
\hline
\end{tabular}

\begin{tabular}{|c|c|c|c|}
\hline Compd. & Method & $\mathrm{R}^{1}$ & $\mathrm{Ar}$ \\
\hline 2,12 & $a$ & $\mathrm{Me}$ & \\
\hline 2,13 & $a$ & $\mathrm{Me}$ & \\
\hline 2,14 & $a$ & $\mathrm{Me}$ & \\
\hline 3,15 & $b$ & $\mathrm{MeO}_{2} \mathrm{C}$ & \\
\hline 3,16 & $b$ & $\mathrm{MeO}_{2} \mathrm{C}$ & \\
\hline 4,17 & $c$ & NC & \\
\hline 4,18 & $c$ & NC & \\
\hline
\end{tabular}

Reagents and conditions: (a) appropriate aryl aldehyde (1.25 M equiv.), EtOH, AcOH (catalytic amount), r.t. $3 \mathrm{~h}$, reflux $25 \mathrm{~h}$; (b) appropriate aryl aldehyde (1.33 M equiv.), $\mathrm{MeOH}, \mathrm{AcOH}$ (catalytic amount), r.t. 4 h, reflux 28 h; (c) appropriate aryl aldehyde (1.33 M equiv.), 2-methoxyethanol, $\mathrm{AcOH}$ (catalytic amount), r.t. $2 \mathrm{~h}$, reflux $9 \mathrm{~h}$.

Scheme 2. Synthesis of 6-chloro-7-R ${ }^{1}$-3-(2-arylmethylene-1-methylhydrazino)-1,1-dioxo1,4,2-benzodithiazines 5-18.

Interestingly, compound 16 ( $A r=2,5$-dihydroxyphenyl) exhibited the strongest cytotoxic activity against the $\mathrm{HeLa}$ cell line with an $\mathrm{IC}_{50}$ value of $10 \mu \mathrm{M}$, however shifting of the hydroxyl functionality from position 5 (compound 16) to position 4 (compound 15) on the phenyl ring caused the loss of cytotoxic activity $\left(\mathrm{IC}_{50}=170 \mu \mathrm{M}\right)$ against this cell line. On the other hand, the presence of $A r=5$-nitrothiophene ring (compound 14) determined the highest cytotoxic activities against MCF-7 and HCT-116 cell lines, and affording the $\mathrm{IC}_{50}$ values of $15 \mu \mathrm{M}$ and $16 \mu \mathrm{M}$, respectively, which changed significantly after its replacement by a 5-nitrofuran ring (compound 13) resulting in poor activity ( $\mathrm{IC}_{50}=140 \mu \mathrm{M}, \mathrm{MCF}-7$, and $\mathrm{IC}_{50}=115 \mu \mathrm{M}$, HCT-116).

Nevertheless, considering the compounds' activities against each single cell line, the nature of substituents $\mathrm{R}^{1}$ and $A r$ have varying influences on the biological activity of these compounds. For instance, cytotoxic activity in the series of $N^{2}$-methylenesubstituted 3-hydrazino-7-methyl-1,1-dioxo1,4,2-benzodithiazines against the MCF-7 cell line decreased in the following order: 5-nitrothiophenyl (14) $>$ 2,4-dihydroxyphenyl (6) > pyrrolyl (12) > 3-bromo-5-chloro-2-hydroxyphenyl (11) $\approx 5$-bromo- 
2-hydroxy-3-methoxyphenyl (10) > 5-bromo-2-hydroxyphenyl (8) > 2-hydroxy-5-nitrophenyl (9), suggesting the necessity to choose other and more objective methods of establishing the structure-activity relationships, such as QSAR methodology.

Table 1. Cytotoxicity of compounds 2,4 and 6-18 toward human cancer cell lines.

\begin{tabular}{cccc}
\hline \multirow{2}{*}{ Compounds } & \multicolumn{3}{c}{ IC $_{\mathbf{5 0}}[\boldsymbol{\mu M}]$} \\
\cline { 2 - 4 } & MCF-7 & HCT-116 & HeLa \\
\hline $\mathbf{2}$ & $250 \pm 7$ & $280 \pm 1$ & $125 \pm 7$ \\
$\mathbf{4}$ & $155 \pm 2$ & $240 \pm 5$ & $99 \pm 4$ \\
$\mathbf{6}$ & $45 \pm 0.5$ & $31 \pm 1$ & $66 \pm 2$ \\
$\mathbf{7}$ & $270 \pm 13$ & $150 \pm 4$ & $>>100^{\mathrm{a}}$ \\
$\mathbf{8}$ & $84 \pm 3$ & $38 \pm 1$ & $80 \pm 1$ \\
$\mathbf{9}$ & $95 \pm 1$ & $24 \pm 1$ & $32 \pm 2$ \\
$\mathbf{1 0}$ & $73 \pm 2$ & $39 \pm 1$ & $64 \pm 3$ \\
$\mathbf{1 1}$ & $70 \pm 3$ & $38 \pm 1$ & $75 \pm 3$ \\
$\mathbf{1 2}$ & $63 \pm 1$ & $84 \pm 1$ & $100 \pm 3$ \\
$\mathbf{1 3}$ & $140 \pm 1$ & $115 \pm 2$ & $100 \pm 1$ \\
$\mathbf{1 4}$ & $15 \pm 0.5$ & $16 \pm 0.5$ & $135 \pm 9$ \\
$\mathbf{1 5}$ & $105 \pm 2$ & $70 \pm 2$ & $170 \pm 5$ \\
$\mathbf{1 6}$ & $150 \pm 3$ & $23 \pm 0.5$ & $10 \pm 0.5$ \\
$\mathbf{1 7}$ & $83 \pm 1$ & $74 \pm 1$ & $62 \pm 1$ \\
$\mathbf{1 8}$ & $165 \pm 6$ & $260 \pm 3$ & $140 \pm 7$ \\
Cisplatin & $3.0 \pm 0.1$ & $3.8 \pm 0.2$ & $2.2 \pm 0.1$ \\
\hline${ }^{a}$ viability of HeLa cell line at $100 \mu \mathrm{M}$ of compd $\mathbf{7}$ was $100 \%$.
\end{tabular}

\subsection{Quantitative Structure-Activity Relationships (QSARs) of Cytotoxic Activity}

QSAR analysis was performed in order to correlate the cytotoxic activity of the 1,1-dioxo-1,4,2benzodithiazine derivatives with their chemical structure and to determine the most important parameters controlling their pharmacological effects. The optimized structures of tested compounds were sketched using the geometry optimization function of Spartan software (Spartan '08, Wavefunction, Inc., Irvine, CA, USA) [20]. The electronic, steric, hydrophilic and hydrophobic features of molecules obtained from molecular modelling descriptors (Tables 2 and 3) have been applied to the statistical calculations. The QSAR models for each cell line were developed separately. Compound 7 was excluded from the data set of QSAR analysis because of very low cytotoxic activity.

The QSAR model generation was done using the Statistica package (v.10, Statsoft, Tulsa, OK, USA) [21], and to search for optimal QSAR models multiple linear regression (MLR) along with stepwise algorithm were employed. In order to improve the statistical performance of the HCT-116 model, compounds $\mathbf{1 2}$ and $\mathbf{1 8}$ were removed from dataset. Both compounds were identified as outliers based on the initial experimental versus predicted activity plot. A much higher difference between experimental and predicted activity in comparison to the rest of compounds, suggests that these compounds possess some additional features explaining their biological activity, that was not covered in the applied descriptor dataset. 
Table 2. Theoretical molecular parameters of compounds 6, 8-18: CPK Area, CPK Volume, PSA, MW, CFD (HBD), CFD (HBA), Angle (i, j, k), Dihedral (i, j, k, l), Distance (i, j), LogP, Hardness.

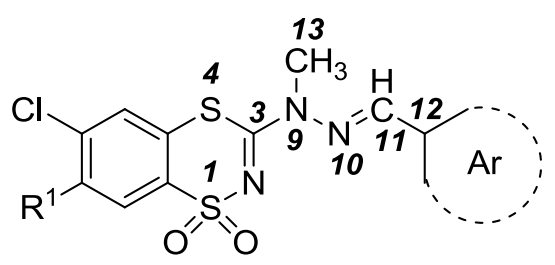

\begin{tabular}{|c|c|c|c|c|c|c|c|c|c|c|c|c|c|c|}
\hline Compd & $\begin{array}{c}\text { CPK Area } \\
\left(\AA^{2}\right)\end{array}$ & $\begin{array}{c}\text { CPK Volume } \\
\qquad\left(\AA^{3}\right)\end{array}$ & $\operatorname{PSA}\left(\AA^{2}\right)$ & $\begin{array}{c}\text { MW } \\
\text { (amu) }\end{array}$ & $\begin{array}{c}\text { CFD } \\
\text { (HBD) }\end{array}$ & $\begin{array}{c}\text { CFD } \\
\text { (HBA) }\end{array}$ & $\begin{array}{c}\text { Angle } \\
\text { (C3,N9,N10) }\end{array}$ & $\begin{array}{c}\text { Angle } \\
\text { (N9,N10,C11) }\end{array}$ & $\begin{array}{c}\text { Dihedral } \\
\text { (C3,N9,N10,C11) }\end{array}$ & $\begin{array}{c}\text { Dihedral } \\
\text { (N9,N10,C11,C12) }\end{array}$ & $\begin{array}{l}\text { Distance } \\
\text { (S4,C12) }\end{array}$ & $\begin{array}{l}\text { Distance } \\
\text { (S1,C12) }\end{array}$ & $\log P$ & Hardness \\
\hline 6 & 378.32 & 351.11 & 89.6827 & 411.890 & 2 & 8 & 119.59 & 115.50 & 69.29 & -179.26 & 6.053 & 5.332 & 4.8599 & 0.199531 \\
\hline 8 & 389.58 & 362.09 & 69.9921 & 474.787 & 1 & 7 & 119.89 & 116.30 & 65.86 & -179.35 & 6.053 & 5.325 & 6.0783 & 0.197595 \\
\hline 9 & 395.72 & 366.01 & 109.1826 & 440.888 & 1 & 10 & 120.39 & 117.25 & 61.89 & -179.55 & 6.052 & 5.320 & 5.2833 & 0.201345 \\
\hline 10 & 417.97 & 388.93 & 74.8555 & 504.813 & 1 & 8 & 119.77 & 116.17 & 66.77 & -179.57 & 6.055 & 5.335 & 5.9519 & 0.195736 \\
\hline 11 & 402.80 & 375.17 & 68.4649 & 509.232 & 1 & 7 & 119.73 & 116.36 & 65.77 & -179.05 & 6.050 & 5.317 & 6.6365 & 0.197948 \\
\hline 12 & 346.63 & 318.50 & 63.7233 & 368.869 & 1 & 7 & 121.27 & 117.32 & 59.23 & 179.13 & 6.044 & 5.315 & 2.9324 & 0.190920 \\
\hline 13 & 370.32 & 337.44 & 99.2438 & 414.850 & 0 & 10 & 120.74 & 118.17 & 58.08 & -179.47 & 6.027 & 5.308 & 3.6225 & 0.193076 \\
\hline 14 & 377.41 & 345.96 & 91.1344 & 430.917 & 0 & 10 & 121.28 & 118.67 & 55.91 & 179.99 & 6.045 & 5.329 & 4.3443 & 0.186695 \\
\hline 15 & 411.87 & 380.93 & 110.6991 & 455.899 & 2 & 9 & 119.50 & 115.35 & 69.58 & -179.19 & 6.053 & 5.334 & 4.1929 & 0.188761 \\
\hline 16 & 411.80 & 380.88 & 110.6926 & 455.899 & 2 & 9 & 119.90 & 116.04 & 66.55 & -179.41 & 6.058 & 5.337 & 4.1929 & 0.184929 \\
\hline 17 & 370.21 & 345.35 & 85.0143 & 406.874 & 1 & 8 & 119.29 & 115.11 & 63.61 & -177.70 & 6.017 & 5.199 & 4.7956 & 0.185247 \\
\hline 18 & 371.53 & 338.82 & 114.5075 & 425.833 & 0 & 11 & 120.30 & 117.52 & 60.87 & -179.34 & 6.027 & 5.303 & 3.1687 & 0.194908 \\
\hline
\end{tabular}

CPK Area-surface area of a space-filling model; CPK Volume-volume of a space-filling model; PSA—polar surface area (N, O + attached hydrogens); MW - molecular weight; CFD (HBD) - number of hydrogen bond donors; CFD (HBA)—number of hydrogen bond acceptors; Angle (i, j, k) - angle involving atoms i, j, k (degrees); Dihedral (i, j, k, l)—dihedral angle involving atoms i, j, k, 1 (degrees); Distance (i, j)—distance involving atoms i, j ( $\AA$ ); LogP-lipophilicity estimated from Crippen model [22]; Hardness--(HOMO-LUMO)/2 (eV). 
Table 3. Theoretical molecular parameters of compounds 6, 8-18: E, E HOMO, E LUMO, Elect (i), Mull (i), Nat (i), Electronegativity.

\begin{tabular}{|c|c|c|c|c|c|c|c|c|c|c|c|c|c|c|}
\hline \multirow{2}{*}{ Compd } & $\mathbf{E}$ & E HOMO & E LUMO & LUMO-HOMO & \multirow{2}{*}{$\begin{array}{l}\text { Elect } \\
\text { (C13) }\end{array}$} & \multirow{2}{*}{$\begin{array}{l}\text { Mull } \\
\text { (C13) }\end{array}$} & \multirow{2}{*}{$\begin{array}{c}\text { Nat } \\
\text { (C13) }\end{array}$} & \multirow{2}{*}{$\begin{array}{l}\text { Elect } \\
\text { (C11) }\end{array}$} & \multirow{2}{*}{$\begin{array}{l}\text { Mull } \\
\text { (C11) }\end{array}$} & \multirow{2}{*}{$\begin{array}{l}\text { Nat } \\
\text { (C11) }\end{array}$} & \multirow{2}{*}{$\begin{array}{l}\text { Elect } \\
\text { (N10) }\end{array}$} & \multirow{2}{*}{$\begin{array}{l}\text { Mull } \\
\text { (N10) }\end{array}$} & \multirow{2}{*}{$\begin{array}{c}\text { Nat } \\
\text { (N10) }\end{array}$} & \multirow{2}{*}{ Electronegativity } \\
\hline & & & $\mathbf{c a l} / \mathbf{m o l}$ ) & & & & & & & & & & & \\
\hline 6 & -1462850 & -194.26 & 56.16 & 250.42 & -0.579 & -0.303 & -0.410 & 0.330 & 0.224 & 0.226 & -0.384 & -0.352 & -0.376 & 0.110038 \\
\hline 8 & -3029531 & -201.90 & 46.09 & 247.99 & -0.608 & -0.306 & -0.411 & 0.299 & 0.217 & 0.213 & -0.365 & -0.338 & -0.355 & 0.124147 \\
\hline 10 & -3100993 & -196.45 & 49.20 & 245.65 & -0.587 & -0.306 & -0.411 & 0.297 & 0.217 & 0.213 & -0.355 & -0.337 & -0.353 & 0.117330 \\
\hline 11 & -3317493 & -208.48 & 39.95 & 248.43 & -0.582 & -0.307 & -0.411 & 0.301 & 0.218 & 0.209 & -0.350 & -0.334 & -0.349 & 0.134281 \\
\hline 12 & -1355166 & -186.14 & 53.47 & 239.61 & -0.662 & -0.306 & -0.410 & 0.175 & 0.157 & 0.183 & -0.384 & -0.327 & -0.351 & 0.105707 \\
\hline 15 & -1580568 & -195.25 & 41.65 & 236.90 & -0.595 & -0.304 & -0.411 & 0.350 & 0.225 & 0.228 & -0.386 & -0.351 & -0.377 & 0.122384 \\
\hline 16 & -1580564 & -191.14 & 40.95 & 232.09 & -0.574 & -0.307 & -0.411 & 0.347 & 0.212 & 0.215 & -0.375 & -0.334 & -0.355 & 0.119665 \\
\hline 17 & -1448937 & -206.78 & 25.71 & 232.49 & -0.575 & -0.307 & -0.411 & 0.326 & 0.158 & 0.208 & -0.399 & -0.310 & -0.350 & 0.144282 \\
\hline 18 & -1528335 & -224.84 & 19.78 & 244.62 & -0.595 & -0.313 & -0.413 & 0.150 & 0.139 & 0.150 & -0.383 & -0.296 & -0.316 & 0.163393 \\
\hline
\end{tabular}

E - total energy; E HOMO - energy of highest-occupied molecular orbital; E LUMO - energy of lowest-occupied molecular orbital; Elect (i) —electrostatic charge on atom i; Mull (i)—Mulliken charge on atom i; Nat (i)—natural charge on atom i; Electronegativity—-(HOMO + LUMO)/2 (eV). 
QSAR models were validated using the leave-one-out cross validation technique. In the case of the quantitative structure-activity relationships for the HeLa cell line a suitable statistical model was not found.

Details of the predictive performance of constructed QSAR models are shown in Table 4. Predicted IC50 values were described by equations as the function of significant descriptor values. The observed as well as the predicted cytotoxic activities are given in Table 5. The statistical significance of equations, as well as high $R_{c v}$ value and value of RMSECV comparable to s, suggests that the obtained QSAR model can be used to explain relationships between chemical structure and activity.

Table 4. The QSAR equations and their predictive performance in predicting cytotoxic activity of the 1,1-dioxo-1,4,2-benzodithiazine derivatives against MCF-7 and HCT-116 cell lines.

\begin{tabular}{|c|c|c|c|c|c|c|c|c|}
\hline Cell Line & Equation & $\mathbf{N}$ & $\mathbf{R}$ & $\mathbf{s}$ & $\mathbf{R}_{\mathrm{cv}}$ & RMSECV & $\mathbf{F}$ & $\mathbf{p}$ \\
\hline MCF-7 & $\begin{array}{l}\mathrm{IC}_{50}=-72361.3(\text { Nat C13) } \\
+2.8(\mathrm{E} \text { HOMO })-29088.3\end{array}$ & 12 & 0.874 & 23.52 & 0.750 & 28.66 & 14.592 & 0.001499 \\
\hline НСТ-116 & $\begin{array}{l}\mathrm{IC}_{50}=-45749.1(\mathrm{Nat} C 13) \\
-937.3(\text { Elect N10) }-19099.8\end{array}$ & 10 & 0.902 & 14.97 & 0.715 & 21.54 & 15.303 & 0.002782 \\
\hline
\end{tabular}

$\mathrm{N}$-number of compounds in data set; $\mathrm{R}-\mathrm{a}$ correlation coefficient; $\mathrm{s}-\mathrm{a}$ standard error of estimate; $\mathrm{R}_{\mathrm{cv}}$ - a correlation coefficient of leave-one-out cross validation (LOO-CV); RMSECV — a root mean square error LOO-CV; F-Fisher test value; $\mathrm{p}$ - significance level of $\mathrm{F}$ test.

Table 5. The cytotoxic activity against MCF-7 and HCT-116 obtained from experiments (observed) and from statistical calculations (predicted).

\begin{tabular}{ccccc}
\hline \multirow{3}{*}{ Compounds } & \multicolumn{4}{c}{ IC $_{\mathbf{5 0}}[\boldsymbol{\mu M}]$} \\
\cline { 2 - 5 } & Observed & Predicted & Observed & Predicted \\
\cline { 2 - 5 } $\mathbf{6}$ & $45 \pm 0.5$ & 41 & $31 \pm 1$ & 17 \\
$\mathbf{8}$ & $84 \pm 3$ & 92 & $38 \pm 1$ & 45 \\
$\mathbf{9}$ & $95 \pm 1$ & 57 & $24 \pm 1$ & 20 \\
$\mathbf{1 0}$ & $73 \pm 2$ & 108 & $39 \pm 1$ & 36 \\
$\mathbf{1 1}$ & $70 \pm 3$ & 74 & $38 \pm 1$ & 31 \\
$\mathbf{1 2}$ & $63 \pm 1$ & 64 & $84 \pm 1$ & $-{ }^{\mathrm{a}}$ \\
$\mathbf{1 3}$ & $140 \pm 1$ & 120 & $115 \pm 2$ & 101 \\
$\mathbf{1 4}$ & $15 \pm 0.5$ & 46 & $16 \pm 0.5$ & 22 \\
$\mathbf{1 5}$ & $105 \pm 2$ & 111 & $70 \pm 2$ & 65 \\
$\mathbf{1 6}$ & $150 \pm 3$ & 122 & $23 \pm 0.5$ & 54 \\
$\mathbf{1 7}$ & $83 \pm 1$ & 79 & $74 \pm 1$ & 77 \\
$\mathbf{1 8}$ & $165 \pm 6$ & 174 & $260 \pm 3$ & $-{ }^{\mathrm{a}}$ \\
\hline & & & & \\
& & & & \\
\end{tabular}

The statistical MCF-7 model indicated that natural charge on carbon atom C13 and energy of highest occupied molecular orbital (HOMO) are highly involved in cytotoxic activity against MCF-7 cell line. It was found that natural charge on carbon atom $\mathrm{C} 13$ has a negative weight in the correlation, whereas energy of HOMO positively correlates with cytotoxic activity of compounds. The significant influence of electronic properties of atom $\mathrm{C} 13$ on biological activity has been illustrated by optimized structures including marked natural charges and isosurface plots of HOMO, that show the energy level of HOMO 
(Figure 2). The anticancer properties of compounds 6 and 12 have been determined by low natural charge of C13 in spite of low HOMO energy. Apparently differences in charges of C13 had the influence on totally different biological activities of compounds 13 and 14, despite their similar HOMO energy.
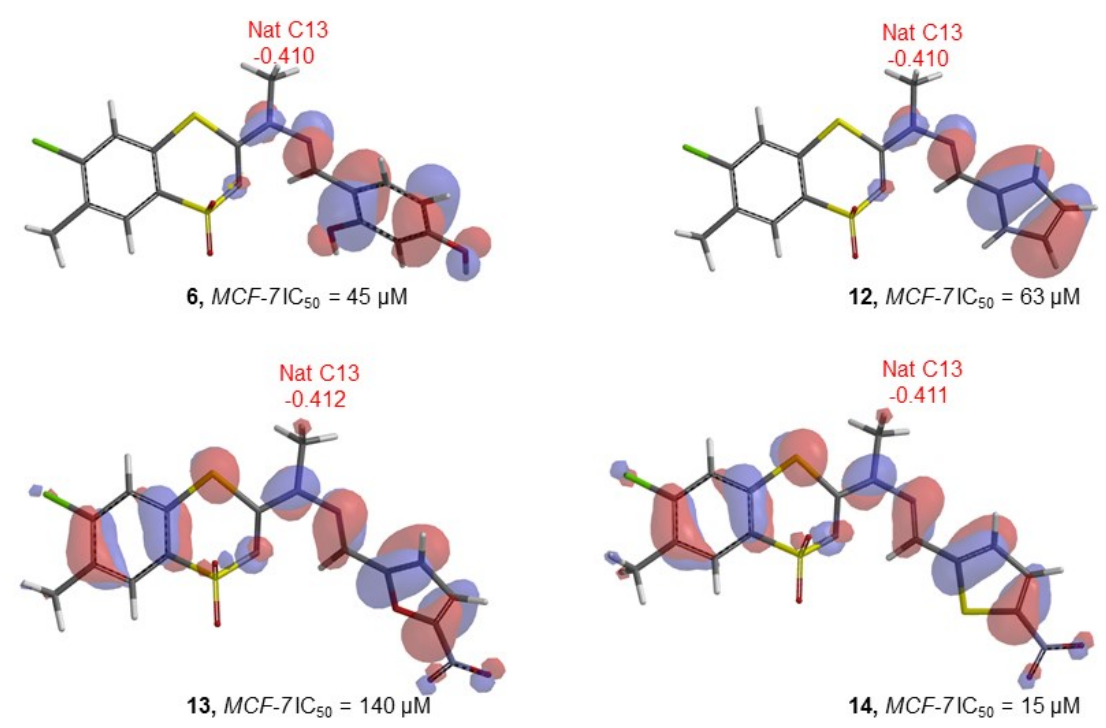

Figure 2. Isosurface plots of HOMO of compounds 6, 12, 13 and 14, and natural charges on atom $\mathrm{C} 13$.

As shown the QSAR model for HCT-116 cell line, natural charge on carbon atom C13 as well as electrostatic charge on nitrogen atom N10 correlates with cytotoxic activity of compound. According to the equation, high anticancer activity is affected by low natural charge of $\mathrm{C} 13$ as the most important descriptor and low electrostatic charge of N10. As indicated in Table 3 inactive compounds exhibited relatively larger natural charges on $\mathrm{C} 13$ atom.

The QSAR results indicate that the cytotoxic activities of 1,1-dioxo-1,4,2-benzodithiazines against MCF-7 and HCT-116 are related to their molecular structure and especially the nature of the $A r$ group. Taken together the SAR and QSAR results on the cytotoxic activities of these analogs may provide valuable information for the further design of novel anticancer agents.

\section{Experimental Section}

\subsection{General Information}

The melting points were determined on a Boethius PHMK apparatus and are uncorrected. Infrared (IR) spectra were recorded on a Thermo Mattson Satellite FTIR spectrophotometer. The NMR spectra were recorded on a Varian Gemini 200 spectrometer at $200 \mathrm{MHz}\left({ }^{1} \mathrm{H}-\mathrm{NMR}\right)$ or on a Varian Unity 500 Plus apparatus at $500 \mathrm{MHz}\left({ }^{1} \mathrm{H}-\mathrm{NMR}\right)$ and $125 \mathrm{MHz}\left({ }^{13} \mathrm{C}-\mathrm{NMR}\right)$. Chemical shifts are expressed as $\delta$ values in parts per million (ppm) relative to TMS as an internal standard. Spectra were acquired in deuterated dimethyl sulfoxide (DMSO- $d_{6}$ ). Elemental analyses were performed on PerkinElmer 2400 Series II CHN Elemental Analyzer and were in agreement with the theoretical values within $\pm 0.4 \%$ range. The commercially unavailable new substrates were obtained according to the following methods described previously: 1a [23], 1b [24] and 1c [25]. 


\subsection{Chemistry}

3.2.1. Procedure for the Preparation of $N$-Methyl- $N$-(6-chloro-7-R ${ }^{1}-1,1-$ dioxo-1,4,2-benzodithiazin-3yl)hydrazines 2-4

A mixture of methylhydrazine $(2.39 \mathrm{~g}, 0.052 \mathrm{~mol})$ and the corresponding 3-methylthio-1,4,2benzodithiazine 1a-c $(0.05 \mathrm{~mol})$ in dry acetonitrile $(60 \mathrm{~mL})$ was stirred at room temperature for $8 \mathrm{~h}$, followed by reflux until the evolution of MeSH had ceased (10-16 h) (Caution: because of its high toxicity, MeSH should be trapped in aqueous $\mathrm{NaOH}$ solution). After cooling to room temperature the reaction mixture was stirred for $4 \mathrm{~h}$. The precipitate of the benzodithiazinyl hydrazines obtained was filtered off, washed with acetonitrile $(2 \times 5 \mathrm{~mL})$ and dried. In this manner the following hydrazines were obtained.

N-Methyl-N-(6-chloro-7-methyl-1,1-dioxo-1,4,2-benzodithiazin-3-yl)hydrazine (2). Starting from 6-chloro-7-methyl-3-methylthio-1,1-dioxo-1,4,2-benzoditiazine 1a (14.7 g), the title compound 2 was obtained (13.5 g, 93\%): mp 271-272 ${ }^{\circ} \mathrm{C}$ dec.; IR (KBr) $v_{\max } 3235\left(\mathrm{~N}-\mathrm{NH}_{2}\right), 1645(\mathrm{C}=\mathrm{N}), 1345,1155$ $\left(\mathrm{SO}_{2}\right) \mathrm{cm}^{-1}$; ${ }^{1} \mathrm{H}-\mathrm{NMR}\left(500 \mathrm{MHz}, \mathrm{DMSO}-d_{6}\right) \delta 2.40$ (s, 3H, CH3-7), 3.31 (s, 3H, N-CH3), 5.70 (s, 2H, N-NH 2$), 7.86$ (s, 1H, H-5), 7.92 (s, 1H, H-8) ppm; ${ }^{13} \mathrm{C}-\mathrm{NMR}$ (125 MHz, DMSO-d6) $\delta ~ 19.95,41.37$, 126.66, 128.48, 130.56, 130.59, 137.40, 137.43, 166.82 ppm; anal. C 37.12, H 3.58, N 14.44\% calcd for $\mathrm{C}_{9} \mathrm{H}_{10} \mathrm{ClN}_{3} \mathrm{O}_{2} \mathrm{~S}_{2}, \mathrm{C} 37.04, \mathrm{H} 3.45, \mathrm{~N} 14.40 \%$.

Methyl 6-chloro-3-(1-methylhydrazino)-1,1-dioxo-1,4,2-benzodithiazine-7-carboxylate (3). Starting from 6-chloro-3-methylthio-1,1-dioxo-1,4,2-benzodithiazine-7-carboxylate $\mathbf{1 b}(16 \mathrm{~g})$, the title compound 3 was obtained (15.1 g, 90\%): $\mathrm{mp} 252-253{ }^{\circ} \mathrm{C} \mathrm{dec}$; IR (KBr) $v_{\max } 3360\left(\mathrm{~N}_{-} \mathrm{NH}_{2}\right), 1740(\mathrm{C}=\mathrm{O}), 1340$, $1155\left(\mathrm{SO}_{2}\right) \mathrm{cm}^{-1}$; ${ }^{1} \mathrm{H}-\mathrm{NMR}\left(500 \mathrm{MHz}, \mathrm{DMSO}-d_{6}\right) \delta 3.30\left(\mathrm{~s}, 3 \mathrm{H}, \mathrm{N}-\mathrm{CH}_{3}\right), 3.88\left(\mathrm{~s}, 3 \mathrm{H}, \mathrm{CH}_{3} \mathrm{O}\right), 5.78(\mathrm{~s}$, $2 \mathrm{H}, \mathrm{N}-\mathrm{NH}_{2}$ ), 8.09 (s, 1H, H-5), 8.29 (s, $1 \mathrm{H}, \mathrm{H}-8$ ) ppm; ${ }^{13} \mathrm{C}-\mathrm{NMR}$ (125 MHz, DMSO-d6) $\delta 41.48,53.68$, $127.17,129.90,130.41,130.88,135.73,137.28,164.25,166.17$ ppm; anal. C 35.72, H 3.10, N 12.57\% calcd for $\mathrm{C}_{10} \mathrm{H}_{10} \mathrm{Cl} \mathrm{N}_{3} \mathrm{O}_{4} \mathrm{~S}_{2}, \mathrm{C} 35.77, \mathrm{H} 3.00, \mathrm{~N} 12.51 \%$.

N-Methyl-N-(6-chloro-7-cyano-1,1-dioxo-1,4,2-benzodithiazin-3-yl)hydrazine (4). Starting from 6-chloro-7-cyano-3-methylthio-1,1-dioxo-1,4,2-benzodithiazine 1c (15.2 g), the title compound 4 was obtained (13.7 g, 91\%): mp 285-286 ${ }^{\circ} \mathrm{C}$ dec.; IR (KBr) $v_{\max } 3320,2245\left(\mathrm{~N}-\mathrm{NH}_{2}\right), 2235(\mathrm{C} \equiv \mathrm{N}), 1340$, $1155\left(\mathrm{SO}_{2}\right) \mathrm{cm}^{-1}$; ${ }^{1} \mathrm{H}-\mathrm{NMR}\left(200 \mathrm{MHz}, \mathrm{DMSO}-d_{6}\right) \delta 3.35$ (s, 3H, N-CH$), 5.82\left(\mathrm{~s}, 2 \mathrm{H}, \mathrm{N}-\mathrm{NH}_{2}\right), 8.27$ (s, $1 \mathrm{H}, \mathrm{H}-5), 8.50$ (s, $1 \mathrm{H}, \mathrm{H}-8) \mathrm{ppm} ;{ }^{13} \mathrm{C}-\mathrm{NMR}\left(125 \mathrm{MHz}, \mathrm{DMSO}-d_{6}\right) \delta 41.52,112.64,115.41,129.95$, 130.53, 130.98, 138.70, 139.43, 165.96 ppm; anal. C 35.70, H 2.40, N 18.52\% calcd for $\mathrm{C}_{9} \mathrm{H}_{7} \mathrm{Cl} \mathrm{N}_{4} \mathrm{O}_{2} \mathrm{~S}_{2}$, C 35.70, H 2.33, N $18.50 \%$.

3.2.2. General Procedure for the Preparation of 6-Chloro-7-methyl-3-(2-arylmethylene-1-methylhydrazino)-1,1-dioxo-1,4,2-benzodithiazines 5-14

A mixture of the benzodithiazinyl hydrazine $2(1.17 \mathrm{~g}, 4 \mathrm{mmol})$, the appropriate aryl carbaldehyde $(5 \mathrm{mmol})$ and glacial acetic acid $(0.5 \mathrm{~mL}$, catalytic amount $)$ in ethanol $(25 \mathrm{~mL})$ was stirred at room temperature for $3 \mathrm{~h}$, followed at reflux for $25 \mathrm{~h}$. After cooling to room temperature and standing overnight, the precipitate was filtered off, washed with ethanol $(4 \times 2 \mathrm{~mL})$ and dried. In this manner the following benzodithiazines were obtained. 
6-Chloro-7-methyl-3-[2-(hydroxybenzylidene)-1-methylhydrazino]-1,1-dioxo-1,4,2-benzo-dithiazine) (5). Starting from 2-hydroxybenzaldehyde $(0.61 \mathrm{~g})$, the title compound 5 was obtained $(1.45 \mathrm{~g}, 92 \%)$ : $\mathrm{mp}$ 313-315 ${ }^{\circ} \mathrm{C}$ dec.; IR (KBr) $v_{\max } 3225(\mathrm{OH}), 1340,1160\left(\mathrm{SO}_{2}\right) \mathrm{cm}^{-1} ;{ }^{1} \mathrm{H}-\mathrm{NMR}\left(200 \mathrm{MHz}, \mathrm{DMSO}-d_{6}\right) \delta$ 2.44 (s, 3H, $\left.\mathrm{CH}_{3}-7\right), 3.66$ (s, 3H, N-CH3), 6.91-7.00 (m, 2H, Ph), 7.31-7.40 (m, 1H, Ph), 7.85 (dd, $\left.J_{\text {ortho }}=7.7 \mathrm{~Hz}, J_{\text {meta }}=1.6 \mathrm{~Hz}, 1 \mathrm{H}, \mathrm{H}-3, \mathrm{Ph}\right), 8.03$ (s, 2H, H-5 and H-8, benzodithiazine), 8.45 (s, 1H, $\mathrm{N}=\mathrm{CH}$ ), 10.35 (br.s, $1 \mathrm{H}, \mathrm{OH}$ ) ppm; anal. C 48.60, H 3.61, N 10.60\% calcd for $\mathrm{C}_{16} \mathrm{H}_{14} \mathrm{ClN}_{3} \mathrm{O}_{3} \mathrm{~S}_{2}, \mathrm{C} 48.54$, H 3.56, N 10.61\%.

6-Chloro-7-methyl-3-[2-(2,4-dihydroxybenzylidene-1-methylhydrazino]-1,1-dioxo-1,4,2-benzodithiazine (6). Starting from 2,4-dihydroxybenzaldehyde $(0.69 \mathrm{~g})$, the title compound 6 was obtained $(1.62 \mathrm{~g}, 98 \%)$ : mp 318-319 ${ }^{\circ} \mathrm{C}$ dec.; IR (KBr) $v_{\max } 3395,3310(\mathrm{OH}), 1630(\mathrm{C}=\mathrm{N}), 1340,1310,1165,1150\left(\mathrm{SO}_{2}\right) \mathrm{cm}^{-1}$; ${ }^{1} \mathrm{H}-\mathrm{NMR}\left(200 \mathrm{MHz}, \mathrm{DMSO}-d_{6}\right) \delta 2.45\left(\mathrm{~s}, 3 \mathrm{H}, \mathrm{CH}_{3}-7\right), 3.65$ (s, 3H, N-CH3), 6.38-6.45 (m, 2H, H-3 and $\mathrm{H}-5, \mathrm{Ph}), 7.70$ (d, $J=9.1 \mathrm{~Hz}, 1 \mathrm{H}, \mathrm{H}-6, \mathrm{Ph}), 8.01$ (s, 1H, H-5, benzodithiazine), 8.03 (s, 1H, H-8, benzodithiazine), $8.37(\mathrm{~s}, 1 \mathrm{H}, \mathrm{N}=\mathrm{CH}), 10.10(\mathrm{~s}, 1 \mathrm{H}, \mathrm{OH}-4, \mathrm{Ph}), 10.24$ (s, $1 \mathrm{H}, \mathrm{OH}-2, \mathrm{Ph}) \mathrm{ppm} . ;{ }^{13} \mathrm{C}-\mathrm{NMR}$ $\left(125 \mathrm{MHz}\right.$, DMSO- $\left.d_{6}\right) \delta$ 19.98, 32.73, 103.16, 109.05, 111.67, 126.93, 128.70, 128.90, 129.82, 130.50, 137.87, 138.11, 143.83, 159.78, 162.62, 164.53 ppm; anal. C 46.60, H 3.50, N 10.19\% calcd for $\mathrm{C}_{16} \mathrm{H}_{14} \mathrm{ClN}_{3} \mathrm{O}_{4} \mathrm{~S}_{2}, \mathrm{C} 46.65 \mathrm{H}, 3.42 \mathrm{~N}, 10.20 \%$.

6-Chloro-7-methyl-3[2-(2,5-dihydroxybenzylidene)-1-methylhydrazino]-1,1-dioxo-1,4,2-benzodithiazine (7). Starting from 2,5-dihydroxybenzaldehyde $(0.69 \mathrm{~g})$ the title compound 7 was obtained $(1.60 \mathrm{~g}, 97 \%)$ : mp 314-315 ${ }^{\circ} \mathrm{C}$ dec.; IR (KBr) $v_{\max } 3485(\mathrm{OH}), 3385(\mathrm{OH}), 1615(\mathrm{C}=\mathrm{N}), 1345,1305,1160\left(\mathrm{SO}_{2}\right) \mathrm{cm}^{-1}$; ${ }^{1} \mathrm{H}-\mathrm{NMR}\left(200 \mathrm{MHz}, \mathrm{DMSO}-d_{6}\right) \delta 2.45$ (s, 3H, CH3-7), 3.66 (s, 3H, N-CH3), 6.80 (s 2H, H-3 and H-4, $\mathrm{Ph}$ ), 7.23 (s, 1H, H-6, Ph), 7.98 (s, 1H, H-5, benzodithiazine), 8.04 (s, 1H, H-8, benzodithiazine), 8.39 $(\mathrm{s}, 1 \mathrm{H}, \mathrm{N}=\mathrm{CH}), 9.06$ (s, $1 \mathrm{H}, \mathrm{OH}-5, \mathrm{Ph}), 9.63(\mathrm{~s}, 1 \mathrm{H}, \mathrm{OH}-2, \mathrm{Ph}) \mathrm{ppm} ;{ }^{13} \mathrm{C}-\mathrm{NMR}\left(125 \mathrm{MHz}, \mathrm{DMSO}-d_{6}\right) \delta$ 20.01, 32.71, 111.95, 118.09, 120.12, 121.27, 126.95, 128.58, 129.59, 130.06, 138.10, 138.38, 143.12, 150.64, 151.04, 165.13 ppm; anal. C 45.66, H 3.51, N 10.28\% calcd for $\mathrm{C}_{16} \mathrm{H}_{14} \mathrm{Cl} \mathrm{N}_{3} \mathrm{O}_{4} \mathrm{~S}_{2}, \mathrm{C} 46.65, \mathrm{H}$ 3.42, N $10.20 \%$.

6-Chloro-7-methyl-3-[2-(5-bromo-2-hydroxybenzylidene)-1-methylhydrazino]-1,1-dioxo-1,4,2-

benzodithiazine (8). Starting from 5-bromo-2-hydroxybenzaldehyde $(1.0 \mathrm{~g})$, the title compound 8 was obtained (1.75 g, 92\%): mp 330-331 ${ }^{\circ} \mathrm{C} \mathrm{dec}$.; IR (KBr) vmax $3235(\mathrm{OH}), 1610(\mathrm{C}=\mathrm{N}), 1335,1315,1160$ $\left(\mathrm{SO}_{2}\right) \mathrm{cm}^{-1} ;{ }^{1} \mathrm{H}-\mathrm{NMR}\left(200 \mathrm{MHz}, \mathrm{DMSO}-d_{6}\right) \delta 2.44\left(\mathrm{~s}, 3 \mathrm{H}, \mathrm{CH}_{3}-7\right), 3.66\left(\mathrm{~s}, 3 \mathrm{H}, \mathrm{N}-\mathrm{CH}_{3}\right), 6.94(\mathrm{~d}$, $J=8.7 \mathrm{~Hz}, 1 \mathrm{H}, \mathrm{H}-3, \mathrm{Ph}), 7.50\left(\mathrm{dd}, J_{\text {ortho }}=8.7 \mathrm{~Hz}, J_{\text {meta }}=2.5 \mathrm{~Hz}, 1 \mathrm{H}, \mathrm{H}-4, \mathrm{Ph}\right), 7.88$ (d, $J_{\text {meta }}=2.5 \mathrm{~Hz}$, $1 \mathrm{H}, \mathrm{H}-8, \mathrm{Ph}), 8.03$ (s, 1H, H-5, benzodithiazine), 8.10 (s, 1H, H-8, benzodithiazine), 8.35 (s, $1 \mathrm{H}, \mathrm{N}=\mathrm{CH})$, 10.70 (s, 1H, OH) ppm; anal. C 40.45, H 2.80, N 8.90\% calcd for $\mathrm{C}_{16} \mathrm{H}_{13} \mathrm{BrClN}_{3} \mathrm{O}_{3} \mathrm{~S}_{2}, \mathrm{C} 40.47, \mathrm{H} 2.76$, N 8.85\%.

6-Chloro-7-methyl-3-[2-(2-hydroxy-5-nitrobenzylidene)-1-methylhydrazino]-1,1-dioxo-1,4,2-benzodithiazine (9). Starting from 2-hydroxy-5-nitrobenzaldehyde $(0.84 \mathrm{~g})$, the title compound 9 was obtained (1.68 g, 95\%): $\mathrm{mp} 327-328^{\circ} \mathrm{C}$ dec.; IR (KBr) $v_{\max } 3420(\mathrm{OH}), 1610(\mathrm{C}=\mathrm{N}), 1340,1320,1165\left(\mathrm{SO}_{2}\right) \mathrm{cm}^{-1}$; ${ }^{1} \mathrm{H}-\mathrm{NMR}\left(200 \mathrm{MHz}, \mathrm{DMSO}-d_{6}\right) \delta 2.44\left(\mathrm{~s}, 3 \mathrm{H}, \mathrm{CH}_{3}-7\right), 3.69\left(\mathrm{~s}, 3 \mathrm{H}, \mathrm{N}-\mathrm{CH}_{3}\right), 7.15$ (d, J=9.1 Hz, 1H, $\mathrm{H}-3, \mathrm{Ph}), 8.04$ (s, 2H, H-5 and H-8, benzodithiazine), 8.24 (dd, $J_{\text {ortho }}=9.1 \mathrm{~Hz}, J_{\text {meta }}=2.9 \mathrm{~Hz}, 1 \mathrm{H}, \mathrm{H}-4, \mathrm{Ph}$ ), 
$8.40(\mathrm{~s}, 1 \mathrm{H}, \mathrm{N}=\mathrm{CH}), 8.60\left(\mathrm{~d}, J_{\text {meta }}=2.9 \mathrm{~Hz}, 1 \mathrm{H}, \mathrm{H}-6, \mathrm{Ph}\right), 11.95(\mathrm{~s}, 1 \mathrm{H}, \mathrm{OH})$ ppm; anal. C 43.57, H 3.02, $\mathrm{N} 12.76 \%$ calcd for $\mathrm{C}_{16} \mathrm{H}_{13} \mathrm{ClN}_{4} \mathrm{O}_{5} \mathrm{~S}_{2}, \mathrm{C} 43.58, \mathrm{H} 2.97, \mathrm{~N} 12.70 \%$.

6-Chloro-7-methyl-3-[2-(5-bromo-2-hydroxy-3-methoxybenzylidene)-1-methylhydrazino]-1,1-dioxo1,4,2-benzodithiazine (10). Starting from 5-bromo-2-hydroxy-5-methoxybenzaldehyde (1.16 g), the title compound 10 was obtained (1.95 g, 97\%): $\mathrm{mp} 311-312{ }^{\circ} \mathrm{C} \mathrm{dec}$.; IR (KBr) $v_{\max } 3500(\mathrm{OH}), 1610(\mathrm{C}=\mathrm{N})$, 1350, 1310, $1165\left(\mathrm{SO}_{2}\right) \mathrm{cm}^{-1}$; ${ }^{1} \mathrm{H}-\mathrm{NMR}\left(200 \mathrm{MHz}, \mathrm{DMSO}-d_{6}\right) \delta 2.44$ (s, 3H, CH3-7), $3.66(\mathrm{~s}, 3 \mathrm{H}$, $\left.\mathrm{N}-\mathrm{CH}_{3}\right), 3.88\left(\mathrm{~s}, 3 \mathrm{H}, \mathrm{N}-\mathrm{CH}_{3} \mathrm{O}\right), 7.24\left(\mathrm{~d}, J_{\text {meta }}=1.8 \mathrm{~Hz}, 1 \mathrm{H}, \mathrm{Ph}\right), 7.50\left(\mathrm{~d}, J_{\text {meta }}=1.8 \mathrm{~Hz}, 1 \mathrm{H}, \mathrm{Ph}\right), 8.03(\mathrm{~s}$, $1 \mathrm{H}, \mathrm{H}-5$, benzodithiazine), 8.09 (s, 1H, H-8, benzodithiazine), $8.38(\mathrm{~s}, 1 \mathrm{H}, \mathrm{N}=\mathrm{CH}), 10.01(\mathrm{~s}, 1 \mathrm{H}, \mathrm{OH})$ ppm; anal. C 40.51, H 3.00, N 8.36\% calcd for $\mathrm{C}_{17} \mathrm{H}_{15} \mathrm{BrClN}_{3} \mathrm{O}_{4} \mathrm{~S}_{2}, \mathrm{C} 40.45, \mathrm{H} 2.99$, N 8.32\%.

6-Chloro-7-methyl-3-[2-(3-bromo-5-chloro-2-hydroxybenzylidene)-1-methylhydrazino]-1,1-dioxo1,4,2-benzodithiazine (11). Starting from 3-bromo-5-chloro-2-hydroxybezaldehyde (1.18 g), the title compound 11 was obtained (1.95 g, 93\%): $\mathrm{mp} 337-338^{\circ} \mathrm{C}$ dec.; IR (KBr) $v_{\max } 3425(\mathrm{OH}), 1605(\mathrm{C}=\mathrm{N})$, 1335, 1315, $1165\left(\mathrm{SO}_{2}\right) \mathrm{cm}^{-1}$; ${ }^{1} \mathrm{H}-\mathrm{NMR}\left(200 \mathrm{MHz}, \mathrm{DMSO}-d_{6}\right) \delta 2.45$ (s, 3H, CH $\left.\mathrm{CH}_{3}-7\right), 3.67(\mathrm{~s}, 3 \mathrm{H}$, $\left.\mathrm{N}_{-} \mathrm{CH}_{3}\right), 7.72\left(\mathrm{~d}, J_{\text {meta }}=2.5 \mathrm{~Hz}, 1 \mathrm{H}, \mathrm{Ph}\right), 7.83\left(\mathrm{~d}, J_{\text {meta }}=2.5 \mathrm{~Hz}, 1 \mathrm{H}, \mathrm{Ph}\right), 8.05(\mathrm{~s}, 1 \mathrm{H}, \mathrm{H}-5$, benzodithiazine), 8.11 (s, 1H, H-8, benzodithiazine), 8.47 (s, $1 \mathrm{H}, \mathrm{N}=\mathrm{CH}), 10.40$ (s, $1 \mathrm{H}, \mathrm{OH}) \mathrm{ppm}$; anal. C 37.78, H 2.40, N 8.26\% calcd for $\mathrm{C}_{16} \mathrm{H}_{12} \mathrm{BrCl}_{2} \mathrm{~N}_{3} \mathrm{O}_{3} \mathrm{~S}_{2}, \mathrm{C} 37.73, \mathrm{H} 2.37, \mathrm{~N} 8.25 \%$.

6-Chloro-7-methyl-3-[2-(1H-pyrrol-2-yl)methylene-1-methylhydrazino]-1,1-dioxo-1,4,2-benzodithiazine (12). Starting from $1 H$-pyrrole-2-carbaldehyde $(0.48 \mathrm{~g})$, the title compound 12 was obtained $(1.30 \mathrm{~g}$, 88\%): mp 245-246 ${ }^{\circ} \mathrm{C}$ dec.; IR (KBr) $v_{\max } 3420(\mathrm{NH}), 1615(\mathrm{C}=\mathrm{N}), 1345,1310,1155\left(\mathrm{SO}_{2}\right) \mathrm{cm}^{-1}$; ${ }^{1} \mathrm{H}-\mathrm{NMR}\left(200 \mathrm{MHz}, \mathrm{DMSO}-d_{6}\right) \delta 2.44\left(\mathrm{~s}, 3 \mathrm{H}, \mathrm{CH}_{3}-7\right), 3.62\left(\mathrm{~s}, 3 \mathrm{H}, \mathrm{NCH}_{3}\right), 6.20-6.25(\mathrm{~m}, 1 \mathrm{H}, \mathrm{H}-5$, pyrrole), 6.69 (t, $J=10 \mathrm{~Hz}, 1 \mathrm{H}, \mathrm{H}-4$, pyrrole), 7.10 (s, 1H, H-3, pyrrole), 7.78 (s, 1H, H-5, benzodithiazine), $8.02(\mathrm{~s}, 1 \mathrm{H}, \mathrm{H}-8$, benzodithiazine), $8.21(\mathrm{~s}, 1 \mathrm{H}, \mathrm{N}=\mathrm{CH}), 11.46(\mathrm{~s}, 1 \mathrm{H}, \mathrm{NH}) \mathrm{ppm}$; ${ }^{13} \mathrm{C}-\mathrm{NMR}\left(125 \mathrm{MHz}, \mathrm{DMSO}-d_{6}\right) \delta 19.98,32.95,110.77,116.53,124.83,126.81,126.90,128.35$, $130.12,130.45,137.87,138.12,139.89,164.40$ ppm; anal. C 45.61, H 3.60, N 15.18\% calcd for $\mathrm{C}_{14} \mathrm{H}_{13} \mathrm{ClN}_{4} \mathrm{O}_{2} \mathrm{~S}_{2}, \mathrm{C} 45.58, \mathrm{H} 3.55, \mathrm{~N} 15.19 \%$.

6-Chloro-7-methyl-3-[2-(5-nitrofurfurylidene)-1-methylhydrazino]-1,1-dioxo-1,4,2-benzo-dithiazine (13). Starting from 5-nitrofuran-2-carbaldehyde $(0.7 \mathrm{~g})$, the title compound 13 was obtained $(1.44 \mathrm{~g}, 87 \%)$ : mp 281-282 ${ }^{\circ} \mathrm{C}$ dec.; IR (KBr) $v_{\max } 1615(\mathrm{C}=\mathrm{N}), 1350,1315,1165\left(\mathrm{SO}_{2}\right) \mathrm{cm}^{-1} ;{ }^{1} \mathrm{H}-\mathrm{NMR}(200 \mathrm{MHz}$, DMSO-d6) $\delta 2.45\left(\mathrm{~s}, 3 \mathrm{H}, \mathrm{CH}_{3}-7\right), 3.65\left(\mathrm{~s}, 3 \mathrm{H}, \mathrm{NCH}_{3}\right), 7.31$ (d, $J=3.9 \mathrm{~Hz}, 1 \mathrm{H}$, furan), 7.86 (d, $J=3.9 \mathrm{~Hz}$, $1 \mathrm{H}$, furan), 8.04 (s, $1 \mathrm{H}, \mathrm{H}-5$, benzodithiazine), 8.06 (s, 1H, H-8, benzodithiazine), $8.38(\mathrm{~s}, 1 \mathrm{H}, \mathrm{N}=\mathrm{CH})$ ppm; ${ }^{13} \mathrm{C}-\mathrm{NMR}(125 \mathrm{MHz}$, DMSO-d6) $\delta 20.01,33.26,115.22,117.70,127.10,128.80,129.12,129.44$, 135.18, 138.41, 138.78, 151.16, 152.93, 165.56 ppm; anal. C 40.55, H 2.68, N 13.55\% calcd for $\mathrm{C}_{14} \mathrm{H}_{11} \mathrm{ClN}_{4} \mathrm{O}_{5} \mathrm{~S}_{2}, \mathrm{C} 40.53, \mathrm{H} 2.67, \mathrm{~N} 13.50 \%$.

6-Chloro-7-methyl-3-[2-(5-nitrothiophen-2-ylmethylene)-1-methylhydrazino]-1,1-dioxo-1,4,2-benzodithiazine (14). Starting from 5-nitrothiophene-2-carbaldehyde $(0.79 \mathrm{~g})$, the title compound 14 was obtained (1.50 g, 87\%): $\mathrm{mp} 335-336^{\circ} \mathrm{C}$ dec.; IR $(\mathrm{KBr}) v_{\max } 1600(\mathrm{C}=\mathrm{N}), 1335,1305,1160\left(\mathrm{SO}_{2}\right) \mathrm{cm}^{-1}$; ${ }^{1} \mathrm{H}-\mathrm{NMR}\left(200 \mathrm{MHz}, \mathrm{DMSO}-d_{6}\right) \delta 2.45\left(\mathrm{~s}, 3 \mathrm{H}, \mathrm{CH}_{3}-7\right), 3.65\left(\mathrm{~s}, 3 \mathrm{H}, \mathrm{NCH}_{3}\right), 7.62(\mathrm{~d}, J=4.3 \mathrm{~Hz}, 1 \mathrm{H}$, thiophene), 8.05 (s, 1H, H-5, benzodithiazine), 8.14 (s, 1H, H-8, benzodithiazine), 8.18 (d, J=4.3 Hz, 1H, 
thiophene), $8.62(\mathrm{~s}, 1 \mathrm{H}, \mathrm{N}=\mathrm{CH}) \mathrm{ppm}$; anal. $\mathrm{C} 39.11, \mathrm{H} 2.59, \mathrm{~N} 13.06 \%$ calcd for $\mathrm{C}_{14} \mathrm{H}_{11} \mathrm{ClN}_{4} \mathrm{O}_{4} \mathrm{~S}_{3}, \mathrm{C}$ $39.02, \mathrm{H} 2.57, \mathrm{~N} 13.00 \%$.

3.2.3. Procedure for the Preparation of Methyl 6-Chloro-3-[2-(2,4-dihydroxybenzylidene)-1-methylhydrazino]-1,1-dioxo-1,4,2-benzodithiazine-7-carboxylates 15, 16

A mixture of methyl 6-chloro-3-(1-methylhydrazino)-1,1-dioxo-1,4,2-benzodithiazine-7-carboxylate 3 (1.01 g, $3 \mathrm{mmol})$, and the corresponding 2,4- or 2,5-dihydroxbenzaldehyde $(0.55 \mathrm{~g}, 4 \mathrm{mmol})$ glacial acetic acid $(0.4 \mathrm{~mL}$, catalytic amount) and methanol $(30 \mathrm{~mL})$ was stirred at room temperature for $4 \mathrm{~h}$, followed at reflux for $28 \mathrm{~h}$. After cooling to room temperature and standing overnight, the precipitate was filtered off, washed with methanol $(4 \times 3 \mathrm{~mL})$, and dried. In this manner the following benzodithiazines were obtained.

Methyl 6-chloro-3-[2-(2,4-dihydroxybenzylidene)-1-methylhydrazino]-1,1-dioxo-1,4,2-benzodithiazine7-carboxylate (15). Starting from 2,4-dihydroxybenzaldehyde, the title compound 15 was obtained (1.30 g, 95\%): $\mathrm{mp} 310-311^{\circ} \mathrm{C}$ dec.; IR (KBr) vmax 3320 wide $(\mathrm{OH}), 1715,(\mathrm{C}=\mathrm{O}), 1615(\mathrm{C}=\mathrm{N}), 1330$, 1310, 1150, $1130\left(\mathrm{SO}_{2}\right) \mathrm{cm}^{-1}$; ${ }^{1} \mathrm{H}-\mathrm{NMR}\left(200 \mathrm{MHz}, \mathrm{DMSO}-d_{6}\right) \delta 3.60\left(\mathrm{~s}, 3 \mathrm{H}, \mathrm{N}-\mathrm{CH}_{3}\right), 3.92(\mathrm{~s}, 3 \mathrm{H}$, $\left.\mathrm{CH}_{3} \mathrm{O}-\mathrm{C}=\mathrm{O}\right), 6.37-6.42(\mathrm{~m}, 2 \mathrm{H}, \mathrm{H}-5$ and $\mathrm{H}-6, \mathrm{Ph}), 7.71\left(\mathrm{~d}, J_{\text {ortho }}=9.1 \mathrm{~Hz}, 1 \mathrm{H}, \mathrm{H}-3, \mathrm{Ph}\right), 8.23(\mathrm{~s}, 1 \mathrm{H}$, $\mathrm{H}-5$, benzodithiazine), 8.38 (s, 1H, H-8, benzodithiazine), $8.40(\mathrm{~s}, 1 \mathrm{H}, \mathrm{N}=\mathrm{CH}), 10.15(\mathrm{~s}, 1 \mathrm{H}), 10.25$ (s, 1H, OH) ppm; anal. C 44.78, H 3.12, N 9.26\% calcd for $\mathrm{C}_{17} \mathrm{H}_{14} \mathrm{ClN}_{3} \mathrm{O}_{6} \mathrm{~S}_{2}, \mathrm{C} 44.78, \mathrm{H} 3.09$, N 9.21\%.

Methyl 6-chloro-3-[2-(2,5-dihydroxybenzylidene)-1-methylhydrazino]-1,1-dioxo-1,4,2-benzodithiazine7-carboxylate (16). Starting from 2,5-dihydroxybenzaldehyde, the title compound $\mathbf{1 6}$ was obtained (1.28 g, 94\%): $\mathrm{mp} 303-304{ }^{\circ} \mathrm{C}$ dec.; IR (KBr) $v_{\max } 3420,3310(\mathrm{OH}), 1610(\mathrm{C}=\mathrm{N}), 1340,1305,1170$, $1165\left(\mathrm{SO}_{2}\right) \mathrm{cm}^{-1} ;{ }^{1} \mathrm{H}-\mathrm{NMR}\left(200 \mathrm{MHz}, \mathrm{DMSO}-d_{6}\right) \delta 3.68\left(\mathrm{~s}, 3 \mathrm{H}, \mathrm{N}-\mathrm{CH}_{3}\right), 3.91\left(\mathrm{~s}, 3 \mathrm{H}, \mathrm{CH}_{3} \mathrm{O}-\mathrm{C}=\mathrm{O}\right)$, 6.65-6.85 (m, 2H, H-3 and H-4, Ph), $7.23\left(\mathrm{~d}, J_{\text {meta }}=1.6 \mathrm{~Hz}, 1 \mathrm{H}, \mathrm{H}-6, \mathrm{Ph}\right), 8.21(\mathrm{~s}, 1 \mathrm{H}, \mathrm{H}-5$, benzodithiazine), 8.38 (s, 1H, H-8, benzodithiazine), $8.41(\mathrm{~s}, 1 \mathrm{H}, \mathrm{N}=\mathrm{CH}), 9.09(\mathrm{~s}, 1 \mathrm{H}, \mathrm{OH}), 9.68(\mathrm{~s}, 1 \mathrm{H}$, $\mathrm{OH}) \mathrm{ppm} ;{ }^{13} \mathrm{C}-\mathrm{NMR}(125 \mathrm{MHz}$, DMSO-d 6 ) $\delta 32.99,53.72,112.00,118.12,120.04,121.50,127.55$, $130.26,130.61,131.06,136.22,136.25,143.89,150.74,151.24,164.14,164.35$ ppm; anal. C 44.77, H 3.14, N 9.24\% calcd for $\mathrm{C}_{17} \mathrm{H}_{14} \mathrm{ClN}_{3} \mathrm{O}_{6} \mathrm{~S}_{2}, \mathrm{C} 44.78$, H 3.09, N 9.21\%.

3.2.4. Procedure for the Preparation of 6-Chloro-7-cyano-3-(2-arylmethylene-1-methylhydrazino)-1,1dioxo-1,4,2-benzodithiazines 17, 18

A mixture of $N$-methyl- $N$-(6-chloro-7-cyano-1,1-dioxo-1,4,2-benzodithiazine 4, (0.9 g, $3 \mathrm{mmol}$ ) and the corresponding aryl or heteroaryl carbaldehyde $(4 \mathrm{mmol})$, glacial acetic acid $(0.5 \mathrm{~mL})$ and 2-methoxyethanol $(10 \mathrm{~mL})$ was stirred at room temperature for $2 \mathrm{~h}$, followed at reflux for $9 \mathrm{~h}$. After cooling to room temperature and standing overnight, the precipitate was filtered off, washed with methanol $(5 \times 2 \mathrm{~mL})$, and dried. In this manner the following benzodithiazines were obtained.

6-Chloro-7-cyano-3-[2-hydroxybenzylidene)-1-methylhydrazino]-1,1-dioxo-1,4,2-benzodithiazine (17). Starting from 2-hydroxybenzaldehyde $(0.49 \mathrm{~g})$, the title compound $\mathbf{1 7}$ was obtained $(1.10 \mathrm{~g}, \mathbf{9 0 \%})$ : mp 314-315 ${ }^{\circ} \mathrm{C}$ dec.; IR (KBr) v $v_{\max } 2235(\mathrm{C} \equiv \mathrm{N}), 1605(\mathrm{C}=\mathrm{N}), 1330,1160\left(\mathrm{SO}_{2}\right) \mathrm{cm}^{-1} ;{ }^{1} \mathrm{H}-\mathrm{NMR}$ (200 MHz, DMSO-d6) $\delta 3.69$ (s, 3H, N-CH3), 6.90-7.00 (m, 2H, Ph), 7.33-7.41 (m, 1H, Ph), 7.84 (d, 
$J=7.9 \mathrm{~Hz}, 1 \mathrm{H}, \mathrm{Ph}), 8.41$ (s, 1H, H-5, benzodithiazine), 8.61 (s, 1H, H-8, benzodithiazine), 8.61 (s, 1H,

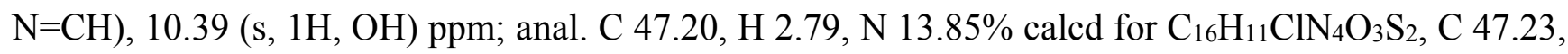
H 2.72, N 13.77\%.

6-Chloro-7-cyano-3-[2-(5-nitrofurfurylidene)-1-methylhydrazino]-1,1-dioxo-1,4,2-benzodithiazine (18). Starting from 5-nitrofuran-2-carbaldehyde (0.56 g), the title compound $\mathbf{1 8}$ was obtained $(1.15 \mathrm{~g}, 90 \%)$ : mp 302-303 ${ }^{\circ} \mathrm{C} \mathrm{dec}$.; IR (KBr) $v_{\max } 2235(\mathrm{C} \equiv \mathrm{N}), 1615(\mathrm{C}=\mathrm{N}), 1360,1325,1165\left(\mathrm{SO}_{2}\right) \mathrm{cm}^{-1}$; ${ }^{1} \mathrm{H}-\mathrm{NMR}$ $\left(\mathrm{DMSO}-d_{6}\right) \delta 3.67$ (s, 3H, N-CH3), 7.34 ( d, $J=3.9 \mathrm{~Hz}, 1 \mathrm{H}$, furan), 7.86 (d, $J=3.9 \mathrm{~Hz}, 1 \mathrm{H}$, furan), 8.43 (s, 1H, H-5, benzodithiazine), $8.44(\mathrm{~s}, 1 \mathrm{H}, \mathrm{H}-8$, benzodithiazine), $8.66(\mathrm{~s}, 1 \mathrm{H}, \mathrm{N}=\mathrm{CH}) \mathrm{ppm}$; ${ }^{13} \mathrm{C}$ NMR $\left(125 \mathrm{MHz}, \mathrm{DMSO}-d_{6}\right) \delta 33.60,113.64,115.18,118.30,130.20,130.41,131.35,136.17$, $137.85,139.37,150.84,153.09,164.56$ ppm; anal. C 39.53, H 1.91, N 16.55\% calcd for $\mathrm{C}_{14} \mathrm{H}_{8} \mathrm{ClN}_{5} \mathrm{O}_{5} \mathrm{~S}_{2}$, C 39.48, H 1.89 , N $16.44 \%$.

\subsection{Cell Culture and Cell Viability Assay}

All chemicals, if not stated otherwise, were obtained from Sigma-Aldrich (St. Louis, MO, USA). The MCF-7 cell line was purchased from Cell Lines Services (Eppelheim, Germany), the HeLa and HCT-116 cell lines were obtained from the Department of Microbiology, Tumor and Cell Biology, Karolinska Institute (Stockholm, Sweden). Cells were cultured in in Dulbecco's modified Eagle's medium (DMEM) supplemented with 10\% fetal bovine serum, $2 \mathrm{mM}$ glutamine, 100 units $/ \mathrm{mL}$ penicillin, and $100 \mu \mathrm{g} / \mathrm{mL}$ streptomycin. Cultures were maintained in a humidified atmosphere with $5 \%$ $\mathrm{CO}_{2}$ at $37^{\circ} \mathrm{C}$ in an incubator (Heraceus, HeraCell).

Cell viability was determined using the 3-(4,5-dimethylthiazol-2-yl)-2,5-diphenyl-tetrazoliumbromide (MTT) assay. Cells were seeded in 96-well plates at a density of $5 \times 10^{3}$ cells/well and treated for $72 \mathrm{~h}$ with the examined compounds in the concentration range $1-100 \mu \mathrm{M}$. Cisplatin was used as a control compound and was examined in the concentration range $0.01-10 \mu \mathrm{M}$. Following treatment, MTT $(0.5 \mathrm{mg} / \mathrm{mL})$ was added to the medium and cells were further incubated for $2 \mathrm{~h}$ at $37^{\circ} \mathrm{C}$. Cells were lysed with DMSO and the absorbance of the formazan solution was measured at $550 \mathrm{~nm}$ with a plate reader (Victor, 1420 multilabel counter). The optical density of the formazan solution was measured at $550 \mathrm{~nm}$ with a plate reader (Victor, 1420 multilabel counter). The experiment was performed in triplicate. Results are expressed as $\mathrm{IC}_{50}$ values. Values are expressed as the mean \pm SD of at least three independent experiments.

\subsection{Molecular Modeling Methodology/Calculations}

Quantum chemical calculations were carried out to study the molecular geometry and electronic structure of 6-chloro-7-R ${ }^{1}$-3-(2-arylmethylene-1-methylhydrazino)-1,1-dioxo-1,4,2-benzodithiazines using the Spartan '08 Software [20]. The full optimized geometries of compounds 6-18 in vacuum were calculated using ab-initio method at the Hartree-Fock (RHF) with 6-31G* polarization basis set.

Multiple linear regression (MLR, along with stepwise algorithm) analysis were performed using Statistica software [21]. The molecular descriptors were used as independent variables. The dependent variable was cytotoxic activity expressed as $\mathrm{IC}_{50}$ values. 
In the leave-one-out cross-validation procedure, a data point (compound) was removed from the analyzed set, the regression was recalculated, and then the predicted value for that point was compared to its observed value. This process was repeated until each datum had been omitted once, and then the sum of squares of these errors for the omitted data were used to calculate the cross-validated root-mean-square error (RMSECV). The RMSECV was calculated using following equation:

$$
R M S E C V=\sqrt{\frac{\sum_{i=1}^{n}\left(y_{i}-\hat{y}_{i}\right)^{2}}{N}}
$$

where $\mathrm{y}_{\mathrm{i}}$ is observed activity, $\hat{y}_{\mathrm{i}}$ is predicted activity, $\mathrm{N}$ is the sample size of the data set.

\section{Conclusions}

We have developed facile methods for the synthesis of new 6-chloro-7-R $\mathrm{R}^{1}-3-(2$-arylmethylene-1methylhydrazino)-1,1-dioxo-1,4,2-benzodithiazines from $N$-methyl- $N$-(6-chloro-7- $\mathrm{R}^{1}$-1,1-dioxo-1,4,2benzodithiazin-3-yl)hydrazines and the appropriate aldehydes. The newly synthesized compounds were tested for their in vitro cytotoxic activity against the MCF-7, HCT-116 and HeLa cell lines. The most active compounds 6 and $\mathbf{9}$ shown the best average cytotoxic activities against all cancer cell lines. Compound 16 exhibited the strongest cytotoxic activity against HeLa cell line with IC50 value of $10 \mu \mathrm{M}$, while 14 was the most cytotoxic compound against MCF-7 and HCT-116 cell lines, giving the IC50 values of $15 \mu \mathrm{M}$ and $16 \mu \mathrm{M}$, respectively. Performed QSAR analysis demonstrate the importance of some electronic properties of molecules in the cytotoxic activities against MCF-7 and HCT-116 cell lines. The QSAR equation showed that a low charge on the $\mathrm{C} 13$ carbon atom and high energy of HOMO are likely required for potent cytotoxic activity against MCF-7 cell line. On the other hand, QSAR HCT-116 model indicated that low natural charge on the $\mathrm{C} 13$ atom and low electrostatic charge on the N10 atom have an impact on the cytotoxic activity of compounds against the HCT-116 cell line. The QSAR results on the cytotoxic activities of the 1,1-dioxo-1,4,2-benzodithiazine analogs will surely provide useful information for the design of new anticancer agents.

\section{Supplementary Materials}

Supplementary materials can be accessed at: http://www.mdpi.com/1420-3049/20/04/5754/s1.

\section{Acknowledgments}

The publishing fee covering the cost to publish in open access was supported by the Ministry of Science and Higher Education of the Republic of Poland, from the quality promoting subsidy under the Leading National Research Centre (KNOW) programme for the years 2012-2017.

\section{Author Contributions}

J.S. and Z.B. created the concept, and designed the study. J.S., B.Ż. and Z.B. performed chemical research and analyzed both the chemical and biological data. J.S. and B.Ż. wrote the manuscript together. A.K. tested the biological activity of the compounds and interpreted the results. B.Z., M.B. and T.B. 
performed statistical analysis and interpreted the statistical models. All authors read and approved the final version of the article.

\section{Conflicts of Interest}

The authors declare no conflict of interest.

\section{References}

1. Brzozowski, Z.; Sławiński, J.; Angielski, S.; Szczepańska-Konkel, M. 1,1-Dioxo-1,4,2benzodithiazine derivatives. III Synthesis and diuretic properties of 3-(R, $\mathrm{R}^{1}$-phenyl)amino-6chloro-7-methyl-1,1-dioxo- 1,4,2-benzodithiazines. Acta Pol. Pharm. 1985, 42, 313-318.

2. Brzozowski, Z.; Sławiński, J. 1,1-Dioxo-1,4,2-benzodithiazine derivatives. IV Synthesis of some novel $N$-substituted 3-amino-6-chloro-7-methyl-1,1-dioxo-1,4,2-benzodithiazines. Acta Pol. Pharm. 1985, 42, 319-325.

3. Brzozowski, Z.; Sławiński, J.; Gajewski, F.; Angielski, S.; Hoppe, A. 1,1-Dioxo-1,4,2-benzodithiazine derivatives. V Synthesis and diuretic properties of some novel 3-phenylamino-7-carboxy-1,1dioxo-1,4,2-benzodithiazines. Acta Pol. Pharm. 1985, 42, 413-420.

4. Brzozowski, Z.; Sławiński, J. Preparation Method for Novel 1,1-Dioxo-3-mercapto-1,4,2benzodithiazines (Sposób otrzymywania nowych 1,1-diokso-3-merkapto-1,4,2-benzoditiazyn). Polish Patent 134567, issued 20 May 1986.

5. Brzozowski, Z.; Sławiński, J.; Gajewski, F.; Angielski, S.; Hoppe, A.; Janiec, W.; Piekarska, T. Preparation Method for Novel 3-Amino-1,1-dioxo-1,4,2-benzodithiazines (Sposób otrzymywania nowych 3-amino-1,1-diokso-1,4,2-benzoditiazyn). Polish Patent 140677, issued 30 April 1988.

6. Brzozowski, Z.; Sławiński, J.; Gajewski, F.; Pomarnacka, E.; Janiec, W.; Piekarska, T. Preparation Method for Novel 3-Amino-1,1-dioxo-1,4,2-benzodithiazine derivatives (Sposób otrzymywania pochodnych 3-amino-1,1-diokso-1,4,2-benzoditiazyny). Polish Patent 141834, issued 30 November 1988.

7. Brzozowski, Z.; Sławiński, J.; Janiec, W.; Cegieła, U.; Śliwiński, L.; Sedlak, I. 1,1-Dioxo-1,4,2benzodithiazine derivatives. XII Synthesis and pharmacological properties of some 6-chloro-3carboxyalkylamino-7-methyl-1,1-dioxo-1,4,2-benzodithiazines. Acta Pol. Pharm. Drug Res. 1992, 49, 75-79.

8. Brzozowski, Z.; Sączewski, F.; Gdaniec, M. Synthesis, structural characterization and in vitro antitumor activity of novel 6-chloro-1,1-dioxo-1,4,2-benzodithiazie derivatives. Bioorg. Med. Chem. 2003, 11, 3673-3681.

9. Brzozowski, Z.; Sączewski, F.; Gdaniec, M. Synthesis, structural characterization and in vitro antitumor activity of 4-dimethylaminopyridinium (6-chloro-1,1-dioxo-1,4,2-benzodithiazin-3yl)methanides. Eur. J. Med. Chem. 2003, 38, 991-999.

10. Brzozowski, Z.; Sączewski, F.; Sławiński, J.; Bednarski, P.J.; Grünert, R.; Gdaniec, M. Synthesis, structural characterization, and in vitro antitumor activity of novel $\mathrm{N}$-(6-chloro-1,1-dioxo-1,4,2benzodithiazin-3-yl)arylsulfonamides. Bioorg. Med. Chem. 2007, 15, 2560-2572. 
11. Brzozowski, Z.; Sączewski, F. A new type of mixed anhydride and its applications to the synthesis of 7-substituted 8-chloro-5,5-dioxoimidazo[1,2-b][1,4,2]benzodithiazines with in vitro antitumor activity. J. Med. Chem. 2002, 45, 430-437.

12. Pomarnacka, E.; Gdaniec, M. Synthesis and anticancer activity of 2-amino-8-chloro-5,5dioxo[1,2,4]triazolo[2,3-b][1,4,2]benzodithiazine derivatives. Bioorg. Med. Chem. 2003, 11, 1259-1267.

13. Brzozowski, Z.; Sączewski, F.; Neamati, N. Synthesis, antitumor and anti-HIV activities of benzodithiazine-dioxides. Bioorg. Med. Chem. 2006, 14, 2985-2993.

14. Brzozowski, Z.; Sączewski, F.; Sanchez, T.; Kuo, C.L.; Gdaniec, M.; Neamati, N. Synthesis, antiviral, and anti-HIV-1 integrase activities of 3-aroyl-1,1-dioxo-1,4,2-benzodithiazines. Bioorg. Med. Chem. 2004, 12, 3663-3672.

15. Brzozowski, Z.; Sączewski, F.; Sławiński, J.; Sanchez, T.; Neamati, N. Synthesis and anti-HIV-1 integrase activities of 3-aroyl-2,3-dihydro-1,1-dioxo-1,4,2-benzodithiazines. Eur. J. Med. Chem. 2009, 44, 190-196.

16. Kubinyi, H. Quantitative structure-activity relationship (QSAR) and molecular modeling in cancer research. J. Cancer Res. Clin. Oncol. 1990, 116, 529-537.

17. Ragnarsson, U. Synthetic methodology for alkyl substituted hydrazines. Chem. Soc. Rev. 2001, 30, 205-213.

18. Schmidt, E.W. Hydrazines and Its Derivatives: Preparation, Properties, Applications, 2nd ed.; VCH: Weinheim, Germany, 2001.

19. Nigst, T.A.; Ammer, J.; Mayr, H. Ambident reactivities of methylhydrazines. Angew. Chem. Int. Ed. 2012, 51, 1353-1356.

20. Spartan '08; Wavefunction, Inc.: Irvine, CA, USA, 2009.

21. Statistica 10; StatSoft. Inc.: Tulsa, OK, USA, 2011.

22. Ghose, A.K.; Pritchett, A.; Crippen, G.M. Atomic Physicochemical Parameters for Three Dimensional Structure Directed Quantitative Structure-Activity Relationships III: Modeling Hydrophobic Interactions. J. Comput. Chem. 1988, 9, 80-90.

23. Brzozowski, Z.; Sławiński, J. 1,1-Dioxo-1,4,2-benzodithiazine derivatives. II Synthesis of some 3mercapto-1,1-dioxo-1,4,2-benzodithiazine derivatives. Acta Pol. Pharm. 1984, 41, 133-139.

24. Brzozowski, Z.; Sławiński, J. 1,1-Dioxo-1,4,2-benzodithiazine derivatives. I Synthesis of some 7-carboxy-3-mercapto-1,1-dioxo-1,4,2-benzodithiazine derivatives. Acta Pol. Pharm. 1984, 41, 5-13.

25. Brzozowski, Z. Derivatives of 2-mercaptobenzenesulphonamide XIV. On the products of selective alcoholysis and hydrolysis of 3-methyltio-1,4,2-benzodithiazine 1,1-dioxide derivatives. Acta Pol. Pharm. Drug Res. 1994, 51, 69-76.

Sample Availability: Samples of the compounds 1-18 are available from the authors.

(C) 2015 by the authors; licensee MDPI, Basel, Switzerland. This article is an open access article distributed under the terms and conditions of the Creative Commons Attribution license (http://creativecommons.org/licenses/by/4.0/). 